\title{
Integral sliding mode fault tolerant control allocation for a class of affine nonlinear system
}

\author{
L. Chen and C. Edwards*and H. Alwi \\ College of Engineering, Mathematics and Physical Sciences, University of Exeter, Exeter, UK.
}

\begin{abstract}
SUMMARY
This paper develops novel fault tolerant integral sliding mode control allocation schemes for a class of over-actuated affine nonlinear system. The proposed schemes rely on an existing baseline controller and the objective is to retain the nominal (fault-free) closed-loop performance in the face of actuator faults/failures by effectively utilizing actuator redundancy. The online control allocation reroutes the control effort to the healthy actuators using knowledge of the actuator effectiveness level estimates. One of the proposed schemes is tested in simulation using a well known high fidelity model of a large civil transport aircraft (B747) from the literature. Good simulation results show the efficacy of the scheme.
\end{abstract}

KEY WORDS: Integral sliding mode; Fault tolerant control; Control allocation

\section{INTRODUCTION}

Sliding mode control schemes have several unique properties, and these have sustained research interest in this area since the 1960s. The most important property is its insensitivity (at least theoretically) to matched uncertainty acting in the control input channels [1, 2, 3]. In conventional first order sliding mode systems, the order of the closed-loop system is reduced compared to the open-loop, by an amount equal to the number of input control signals. The reduced order dynamics during the sliding motion are determined by the choice of sliding surface - which is a key component of the design process. Many different approaches for the design of linear sliding surfaces for uncertain linear systems have been developed, and the area is quite mature [2,3]. In conventional sliding modes the closed-loop behaviour has two quite distinguishable phases: a) the pre-sliding phase in which the system states are driven towards the sliding surface to create a sliding mode; b) the reduced order sliding motion that occurs once the surface is attained. Crucially the insensitivity properties only manifest themselves once sliding has been achieved. Integral sliding modes (ISM) were first discussed in the late 1980s and early 1990s [4, 5]. Two clear distinctions exist between integral sliding modes and conventional first order sliding modes. Firstly, during sliding, ISM systems retain the order of the original system - i.e. no reduction of order occurs (although the property of insensitivity to matched uncertainty is unaffected); secondly there is no reaching phase. Key subsequent work has refined these ideas to combat unmatched uncertainty [6], investigated output feedback formulations [7, 8, 9] and extended these ideas to nonlinear systems [10].

The problem of stabilization of affine nonlinear systems, using variable structure control and sliding mode paradigms, has been widely studied in last decade (e.g. see $[11,12,13,14,10,15])$. One of the earliest works described in [11] transformed the affine nonlinear system (with high relative degree in terms of the system outputs) into one in controllability regular form to achieve output tracking. As argued in [11], a variable structure output feedback control law can be found

$\overline{{ }^{*} \text { Correspondence }}$ to: College of Engineering, Mathematics and Physical Sciences, University of Exeter, Exeter, UK. 
assuming the nonlinear system is decouplable and minimum phase. The work in [12] proposed a stable sliding mode tracking control scheme. Although various variable structure control laws were developed to stabilize affine nonlinear systems $[14,16]$, the occurrence of a sliding mode was often not exploited. Subsequent work in $[17,18,19]$ deals with the design of higher-order sliding mode controllers for a single-input single output (SISO) nonlinear affine system.

The robustness properties of ISM schemes have been exploited to deal with problems associated with affine nonlinear systems using first order sliding concepts [4, 5] and later higher order sliding methods $[20,21]$. General ISM schemes were developed for nonlinear affine systems to minimise the effect of the matched and unmatched uncertainty [10], generalising the results from [6]. The work in [22] proposed a higher order ISM controller for a class of SISO affine nonlinear systems formulated in a nonlinear block controllable form, and this approach achieved finite-time exact compensation of unmatched perturbations with semi-global convergence features, for both regulation and tracking. In [23], an explicit affine nonlinear model predictive control method was combined with an ISM control approach to stabilize nonlinear systems in the presence of additive bounded disturbances, and in [24], an affine nonlinear generalised predictive control method was proposed. The approach includes an ISM framework and guarantees zero steady-state error for a class of affine nonlinear systems in the presence of both matched and unmatched disturbances. In [25], the problem of developing an ISM controller for affine nonlinear systems with input disturbances and unknown nonlinear terms was considered using the adaptive actorcritic control method.

In the last few decades, the subject of fault tolerant control (FTC) has been the focus of significant attention: both theoretically and from an applications perspective (e.g. see [26, 27, 28, 29]). Many methodologies have been explored such as control allocation (CA) [30, 31, 32, 33], physical approaches [34], and $\mathcal{H}_{\infty}$ approaches [35, 36, 37]. (However it is probably fair to say that the most mature are based on linear system representations). During this period, the use of sliding mode controllers as FTC schemes has also been explored [30]. It is at least intuitively clear that certain classes of actuator faults can be modelled as a special case of matched uncertainty. Consequently, for faults which only deteriorate the capabilities of the actuators, sliding mode controllers provide defacto fault tolerance. Integral Sliding Mode control laws have also been researched in this context [38]. However in the literature, very little work has focused on fault tolerant ISM schemes for nonlinear affine systems.

The work in [39] developed a fault tolerant ISM strategy for a class of second order affine nonlinear systems (in a framework similar to an Euler-Lagrange representation). This work was then extended and applied to more general nonlinear affine systems [40]. This paper develops faulttolerant integral sliding mode control allocation (ISM/CA) schemes for a class of nonlinear affine systems. In particular, one version develops an ISM scheme that can be retro-fitted to an existing controller developed using only the primary actuators, to induce robustness with respect to actuator faults/failures, and a CA component to redistribute the control signals to healthy/fault-free actuators based on actuator effectiveness level estimates (which are assumed to be known). To demonstrate the efficacy of the scheme, the retro-fitted ISM/CA procedure will be applied to a longitudinal rigid body aircraft model and evaluated using the RECOVER benchmark [41].

The main contributions of the paper are: i) fault tolerant ISM schemes developed for linear time invariant (LTI) $[42,38]$ and linear parameter varying (LPV) representations [43, 44] are extended into a more general nonlinear affine framework; ii) compared with the work in [10], a control allocation component has been embedded to ensure fault tolerance and the dimension of the switching function reduced; iii) one of the two design schemes developed in this paper introduces fault tolerance by retro-fitting an integral sliding mode control signal to an existing baseline controller in the presence of actuator faults/failures.

The structure of the paper is as follows: Section 2 describes integral sliding mode online control allocation (ISM/CA) schemes created to cope with the presence of actuator faults/failures. In Section 3 , one of the proposed ISM/CA schemes is then applied to a nonlinear longitudinal rigid aircraft model. Concluding remarks are made in Section 4. 


\section{FAULT TOLERANT ISM CONTROL FOR AFFINE NONLINEAR SYSTEMS}

\subsection{Preliminaries}

Consider an over-actuated system subject to faults written in the form

$$
\dot{x}(t)=f(x, t)+g(x) W(t) u(t)+g(x) \zeta_{m}(x, t)
$$

where the state vector $x(t) \in D \subset \mathbb{R}^{n}$ where the compact region $D$ contains the origin. It is assumed that the vector field $f(x, t): D \times \mathbb{R}^{+} \rightarrow \mathbb{R}^{n}$ is known and has at least one equilibrium point which, without loss of generality, occurs at the origin so that $f(0, t)=0$ (after a possible coordinate shift). The signal $u(t) \in \mathbb{R}^{m}$ denotes the system inputs. The last term in (1) represents matched disturbances where the signal $\zeta_{m}(x, t): \mathbb{R}^{n} \times \mathbb{R}^{+} \mapsto \mathbb{R}^{n}$ represents an unknown vector representing modelling uncertainties and external disturbances which is assumed to be worst-casenorm-bounded by a known function $\bar{\zeta}_{m}(x, t)$. In (1), the effectiveness level matrix $W(t) \in \mathbb{R}^{m \times m}$ is a diagonal matrix where the diagonal terms $w_{i}(t) \in\left[\begin{array}{ll}0 & 1\end{array}\right]$. This is a common fault model in the literature [26, 30]. Here it is assumed that a good estimate of $W(t)$ is available from a fault detection and diagnosis (FDD) scheme $[45,46]$ monitoring the system. Suppose the $m$ columns of $g(x): D \rightarrow \mathbb{R}^{n \times m}$ are smooth vector fields and are known. Providing

$$
\epsilon_{0} \leq w_{i}(t) \leq 1 \text { for } i=1 \ldots m
$$

where $0<\epsilon_{0}<1$, then $W(t)$ is nonsingular and the problem of fault tolerant control can be addressed, for example, using the approach in [10]. Furthermore, the associated sliding mode will be unaffected by the faults and uncertainty because both are matched disturbances in equation (1). The situation in which $w_{i}=0$, requiring $\epsilon_{0}=0$, corresponds to the case where the $i$ th actuator completely fails and the corresponding control signal $u_{i}$ does not appear in (1). In this situation, the approach in [10] is no longer directly applicable. (It is perhaps unreasonable to expect nominal performance to be retained in the event of the total failure of certain feedback loops). However, especially in safety critical systems, often physical redundancy is built-in at the design stage to mitigate potential catastrophe. The remainder of the paper considers over-actuated systems which are represented as affine nonlinear systems. The next sections outline the class of systems to be considered and the assumptions made. In what follows two different design approaches (with two different sets of assumptions imposed on $g(x)$ ) will be considered.

\subsection{An ISM Design Approach (Design 1)}

Supposed the input distribution matrix in (1) can be factorized as

$$
g(x)=\tilde{g}(x) N(x)
$$

where $\tilde{g}(x): D \rightarrow \mathbb{R}^{n \times l}$ is smooth and full rank, and the matrix $N(x)$ is also rank $l$ where $l<m$. By assumption the system is over-actuated and $l$ represents the number of controlled outputs required to ascribed to the system appropriate closed-loop performance. Note that the non-unique factorization in (3) must be chosen to avoid an ill-conditioned $N(x)$. Consider the following control allocation scheme to exploit the overactuatedness of the system

$$
u(t)=W(t) N(x)^{T}\left(N(x) W(t)^{2} N(x)^{T}\right)^{-1} v(t)
$$

where $v(t) \in \mathbb{R}^{l}$ represents a virtual control input. The idea is to design the virtual control $v(t)$ to provide appropriate closed loop performance and this signal is distributed to the real physical actuators through (4). Provided the reductions in effectiveness of the actuators and the possibility of total failures modelled by $W(t)$ are such that $\operatorname{det}\left(N(x) W(t)^{2} N(x)^{T}\right) \neq 0$, then the expression in (4) is well defined. From (4) it can be easily shown that $N(x) W(t) u(t)=v(t)$. Then exploiting (3), it follows that (1) can be written as

$$
\dot{x}(t)=f(x, t)+\tilde{g}(x) v(t)+\tilde{g}(x) N(x) \zeta_{m}(x, t)
$$




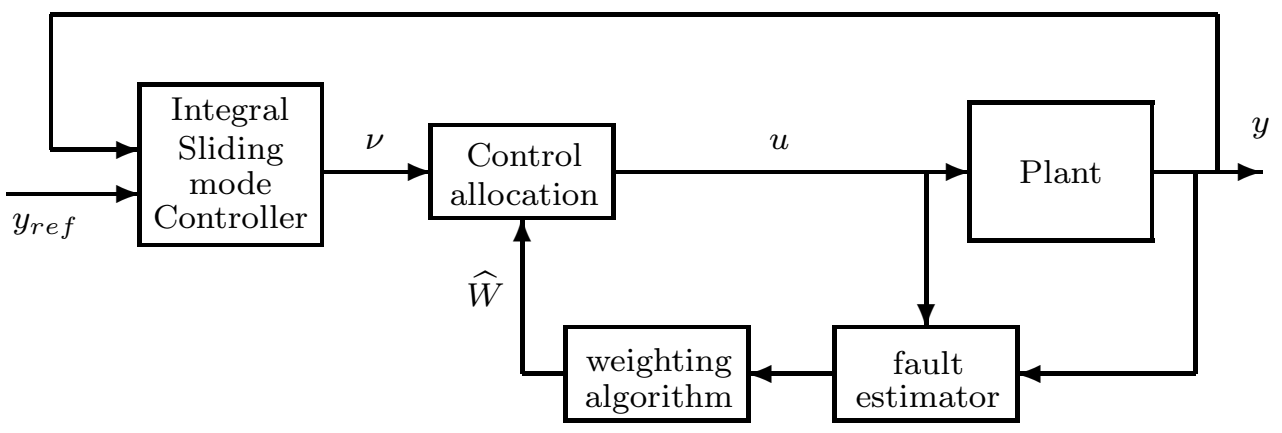

Figure 1. Schematic of the Overall Control Strategy

The control law in (4) depends on $W(t)$, i.e. it requires knowledge of the faults/failures. Assume, as in Fig. 1, a fault diagnosis scheme is available to create an estimate of $W(t)$ denoted by $\widehat{W}(t)$. In the ideal control law (4) replace $W(t)$ with the estimated value $\widehat{W}(t)$ so that

$$
u(t)=\widehat{W}(t) N(x)^{T}\left(N(x) \widehat{W}(t)^{2} N(x)^{T}\right)^{-1} v(t)
$$

Whilst not a perfect estimate of $W(t)$, suppose $\widehat{W}(t)$ satisfies

$$
W(t)=\left(I_{m}-\Delta(t)\right) \widehat{W}(t)
$$

where $\Delta(t) \in \mathbb{R}^{m \times m}$ is a diagonal matrix where $\|\Delta(t)\|<\delta_{\max }<1$. The matrix $\Delta(t)$ is related to the accuracy of estimation, and if $\Delta(t)=0$ then $\widehat{W}(t)=W(t)$. From (7) and noting that $W(t) \in\left[\begin{array}{ll}0 & I\end{array}\right]$, the assumption $\|\Delta(t)\|<\delta_{\max }<1$ ensures the diagonal elements $\hat{w}_{i}(t) \geq 0$ and there can be no change in polarity.

Choose an ISM switching function for the system in (5), as proposed in [10], as

$$
\sigma(x, t)=h(x)-h\left(x_{0}\right)-\int_{t_{0}}^{t} G(x)\left(f(x, \tau)+\tilde{g}(x) N(x) u_{0}(\tau)\right) d \tau
$$

where $u_{0}(t)$ is chosen so that the nominal closed loop system

$$
\dot{x}(t)=f(x, t)+\tilde{g}(x) N(x) u_{0}(t)
$$

is stable and has appropriate performance. The nonlinear function $h(x): \mathbb{R}^{n} \mapsto \mathbb{R}^{m}$ has the property that

$$
G(x):=\frac{\partial h(x)}{\partial x}
$$

and satisfies

$$
\operatorname{rank}(G(x) \tilde{g}(x))=l
$$

The objective is to design a control law so that $\sigma \equiv 0$ for all time, i.e. to ensure a sliding mode is enforced on

$$
\mathcal{S}=\left\{x \in \mathbb{R}^{n}: \sigma(x, t)=0\right\}
$$

It follows from (8) that

$$
\begin{aligned}
\dot{\sigma} & =G(x) \tilde{g}(x) N(x)\left(I_{m}-\Delta(t)\right) \widehat{W}(t) u(x, t)-G(x) \tilde{g}(x) N(x)\left(u_{0}(t)-\zeta_{m}(x, t)\right) \\
& =G(x) \tilde{g}(x) v(x, t)-G(x) \tilde{g}(x) N(x) \Delta(t) N(x)^{\dagger} v(x, t)-G(x) \tilde{g}(x) N(x)\left(u_{0}(t)-\zeta_{m}(x, t)\right)
\end{aligned}
$$

where

$$
N(x)^{\dagger}=\widehat{W}(t)^{2} N(x)^{T}\left(N(x) \widehat{W}(t)^{2} N(x)^{T}\right)^{-1}
$$


Note that $N(x)^{\dagger}$ is a right pseudo inverse of $N(x)$ and by assumption $\operatorname{rank}(N(x))=l$ for all $x \in D$. Consequently, since $\widehat{W}(t)$ is diagonal, $\left\|N(x)^{\dagger}\right\|$ is bounded for any $x \in D$ (for details on this special property of pseudo inverses see $[47,48])$. Define

$$
v(t):=N(x) u_{0}(t)-\rho(x, t) \frac{(G(x) \tilde{g}(x))^{T} \sigma}{\left\|(G(x) \tilde{g}(x))^{T} \sigma\right\|}
$$

where the scalar $\rho(x, t)$ represents the modulation gain which needs to be chosen to ensure sliding can be maintained.

\section{Remark 2.1}

The problem of calculating $u_{0}(t)$ to stabilize affine nonlinear systems such as those in (9) has been widely addressed in the literature. For example, methods such as feedback linearization [49], backstepping [50, 51], methods based on extensions of linear quadratic regulation (LQR) [52], $\mathcal{H}_{\infty}$ (or $\mathcal{L}_{2}$ ) optimal control [53] and sliding mode control [5, 10] have all been applied.

It follows from (13) that

$$
\begin{aligned}
\sigma^{T} \dot{\sigma}= & -\rho(x, t) \frac{\sigma^{T} G(x) \tilde{g}(x)(G(x) \tilde{g}(x))^{T} \sigma}{\left\|(G(x) \tilde{g}(x))^{T} \sigma\right\|}-\sigma^{T} G(x) \tilde{g}(x) N(x) \Delta(t) N(x)^{\dagger} N(x) u_{0} \\
& +\sigma^{T} G(x) \tilde{g}(x) N(x) \Delta(t) N(x)^{\dagger} \rho(x, t) \frac{(G(x) \tilde{g}(x))^{T} \sigma}{\left\|(G(x) \tilde{g}(x))^{T} \sigma\right\|}+\sigma^{T} G(x) \tilde{g}(x) N(x) \zeta_{m}(x, t) \\
\leq & -\rho(x, t)\left\|(G(x) \tilde{g}(x))^{T} \sigma\right\|+\left\|(G(x) \tilde{g}(x))^{T} \sigma\right\|\|N(x)\|^{2}\left\|N(x)^{\dagger}\right\|\left\|u_{0}\right\| \\
& +\rho(x, t) \delta_{\max }\left\|(G(x) \tilde{g}(x))^{T} \sigma\right\|\|N(x)\|\left\|N(x)^{\dagger}\right\|+\left\|(G(x) \tilde{g}(x))^{T} \sigma\right\|\|N(x)\| \bar{\zeta}_{m}(x, t)
\end{aligned}
$$

Here assuming $\delta_{\max }\|N(x)\|\left\|N(x)^{\dagger}\right\|<1$, choose

$$
\rho(x, t)>\frac{\|N(x)\|^{2}\left\|N(x)^{\dagger}\right\|\left\|u_{0}\right\|+\|N(x)\| \bar{\zeta}_{m}(x, t)+\eta_{0}(x, t)}{1-\delta_{\max }\|N(x)\|\left\|N(x)^{\dagger}\right\|}
$$

where $\eta_{0}(x, t)>0$. Provided

$$
\delta_{\max }\|N(x)\|\left\|N(x)^{\dagger}\right\|<1
$$

is satisfied the modulation gain $\rho(x, t)$ is positive.

\section{Remark 2.2}

Note the contribution $\|N(x)\|\left\|N(x)^{\dagger}\right\|$ depends on the plant model and the domain $D$, and the inequality in (18) is effectively a limit on $\delta_{\max }$ which in turn places a constraint in terms of how accurately $\widehat{W}(t)$ must represent $W(t)$.

Using the modulation gain from (17) in inequality (16) it follows

$$
\sigma^{T} \dot{\sigma} \leq-\eta_{0}(x, t) \sqrt{\lambda_{\min }\left(G(x) \tilde{g}(x)(G(x) \tilde{g}(x))^{T}\right)}\|\sigma\|
$$

Inequality (19) represents the reachability condition and guarantees sliding will be maintained despite the occurrence of faults.

From (13), during sliding

$$
0=G(x) \tilde{g}(x) N(x) W(t) u_{e q}(x, t)-G(x) \tilde{g}(x) N(x) u_{0}(t)+G(x) \tilde{g}(x) N(x) \zeta_{m}(x, t)
$$

where the equivalent control signal

$$
u_{e q}(t)=W(t) N(x)^{T}\left(N(x) W(t)^{2} N(x)^{T}\right)^{-1} v_{e q}(t)
$$

and $v_{e q}(t)$ is the equivalent injection signal associated with (15). Since $\operatorname{det}(G(x) g(x)) \neq 0$, equation (20) implies

$$
N(x) W(t) u_{e q}(t)=N(x) u_{0}(t)-N(x) \zeta_{m}(x, t)
$$

Substituting (3) into (1) yields

$$
\dot{x}(t)=f(x, t)+\tilde{g}(x) N(x) W(t) u(t)+\tilde{g}(x) N(x) \zeta_{m}(x, t)
$$


then finally substituting for the equivalent control from (22) yields

$$
\dot{x}(t)=f(x, t)+\tilde{g}(x) N(x) u_{0}(t)
$$

as the dynamics of the sliding motion. From (24) it is clear nominal performance is retained by the appropriate choice of $u_{0}(t)$ despite the faults and uncertainty.

\subsection{A Retro-Fitting Approach (Design 2)}

As an alternative, consider a retro-fitted ISM/CA scheme as shown in Fig. 2. The scheme proposed in Fig. 2 demonstrates the integral sliding mode control signal is retro-fitted to an existing baseline controller to introduce fault tolerance. Compared with the scheme shown in Fig. 1 where the control law is created based on the open-loop plant with no cognizance of any existing controller, the idea of the scheme in Fig, 2 is to use only the primary actuators in the nominal fault-free case (based on an existing controller), and to engage the secondary (redundant) actuators only if faults/failures occur. Now suppose $g(x)$ from (1) has rank $m$ for all $x(t) \in D$ and the system is overactuated. In this subsection, suppose the control input can be written as $u(t)=\left[\begin{array}{ll}u_{p}^{T} & u_{s}^{T}\end{array}\right]^{T} \in \mathbb{R}^{m}$ where the component $u_{p} \in \mathbb{R}^{l}$ represents the primary control inputs and $u_{s} \in \mathbb{R}^{m-l}$ represent the secondary control inputs. In this paper, it is assumed $m \geq 2 l$. Note that this assumption is restrictive but in many over-actuated systems, especially safety critical systems (e.g. civil aircraft), the extent of actuator redundancy is large ${ }^{\dagger}$. Let the input distribution matrix from (1) be conformally partitioned as $g(x)=\left[\begin{array}{ll}g_{p}(x) & g_{s}(x)\end{array}\right]$ where $g_{p}(x): D \rightarrow \mathbb{R}^{n \times l}$ is associated with the primary control inputs and $g_{s}(x): D \rightarrow \mathbb{R}^{n \times(m-l)}$ is associated with the secondary control inputs. For the development which follows partition $W(t)=\operatorname{diag}\left(W_{1}(t), W_{2}(t)\right)$ where $W_{1}(t) \in \mathbb{R}^{l \times l}$ and $W_{2}(t) \in \mathbb{R}^{(m-l) \times(m-l)}$. Here the effectiveness matrices $W_{1}(t)$ and $W_{2}(t)$ are associated with the primary and secondary control inputs, respectively.

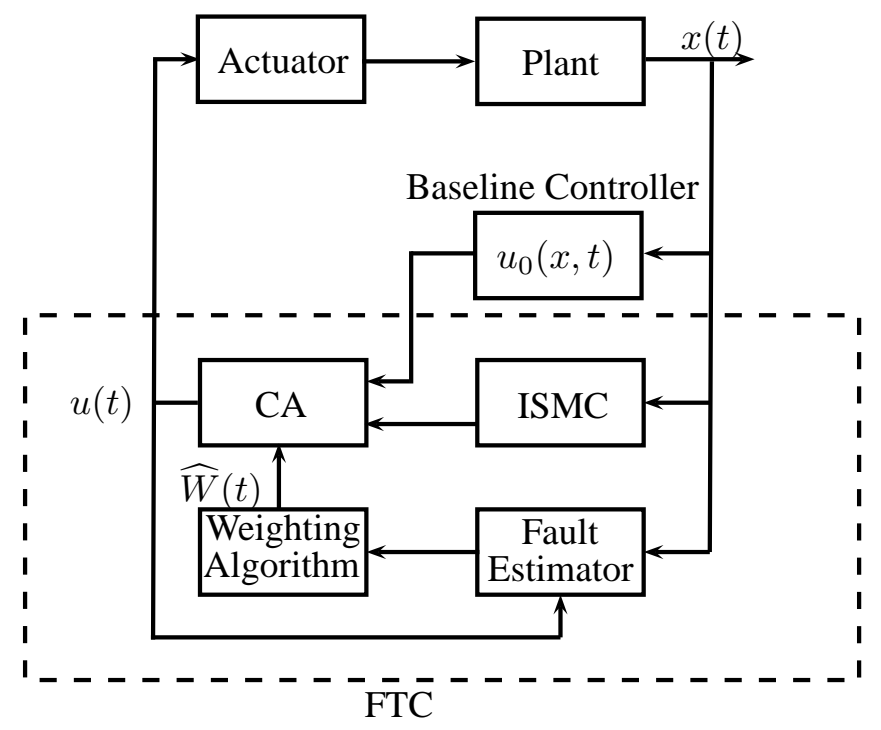

Figure 2. Schematic of the Overall Control Strategy

It follows from the assumptions on $g(x)$ that the $l$ columns of $g_{p}(x)$ are linearly independent vectors for each $x(t) \in D$. Then there exists a diffeomorphism $T(x): D \rightarrow \mathbb{R}^{n}$ such that

$$
\left[L_{g_{p}} T(x)\right]:=\left[\begin{array}{c}
0 \\
\tilde{E}_{21}(x)
\end{array}\right]
$$

\footnotetext{
${ }^{\dagger}$ For example, a Boeing747 has 29 actuator/control surfaces (including the four engines) which could be potentially manipulated independently $[54,41]$.
} 
where $L_{g_{p}} T(x)$ is the Lie Derivative of $T(x)$ with respect to $g_{p}(x)$ and $\tilde{E}_{21}(x) \in \mathbb{R}^{l \times l}$ with $\operatorname{det}\left(\tilde{E}_{21}(x)\right) \neq 0$ for all $x(t) \in D$. Details about $T(x)$ are given in the sequel. Then it follows from the expression in (25)

$$
\left[L_{g} T(x)\right]:=\left[\begin{array}{cc}
0 & \tilde{E}_{12}(x) \\
\tilde{E}_{21}(x) & \tilde{E}_{21}(x) \tilde{E}_{22}(x)
\end{array}\right]
$$

where $L_{g} T(x)$ is the Lie Derivative of $T(x)$ with respect to $g(x), \tilde{E}_{12}(x) \in \mathbb{R}^{(n-l) \times(m-l)}$ and $\tilde{E}_{22}(x) \in \mathbb{R}^{l \times(m-l)}$.

Remark 2.3

According to Frobenius' theorem [55], if $\Xi:=\operatorname{span}\left\{g_{1}, \ldots, g_{l}\right\}$ is involutive, where $g_{1}, \ldots, g_{l}$ are the $l$ columns of $g_{p}(x)$, there always exist smooth functions $\psi_{1}(x), \ldots, \psi_{n-l}(x)$ such that $\left[\partial \psi_{i} / \partial x\right] g_{p}(x)=0$ where $i=1, \ldots, n-l$ for all $x(0) \in D$, and there also exits smooth functions $\psi_{n-l+1}(x), \ldots, \psi_{n}(x)$ such that the diffeomorphism $T(x)=\left[\psi_{1}(x), \ldots, \psi_{n}(x)\right]^{T}$ in a neighborhood of $x(0)$ satisfies (25).

\section{Remark 2.4}

In general, it is not straightforward to create a diffeomorphism $T(x)$ to ensure (25). Nevertheless, the work in [56] for example developed necessary and sufficient conditions for solving $T(x)$ for SISO systems and the work associated with Section 5.4 in [57] extended the conditions to multi-input multi-output systems.

Suppose the uncertainty $\zeta_{m}(x, t)$ in (1) is only associated with the primary control channels. Then applying the system coordinate transformation $T(x): x \rightarrow z$ to equation (1) yields the system representation

$$
\dot{z}(t)=F(z, t)+\underbrace{\left[\begin{array}{cc}
0 & E_{12}(z) \\
E_{21}(z) & E_{21}(z) E_{22}(z)
\end{array}\right]}_{E(z)} W(t) u(t)+\underbrace{\left[\begin{array}{c}
0 \\
E_{21}(z)
\end{array}\right]}_{E_{0}(z)} \zeta_{p}(z, t)
$$

where $E(z)=\tilde{E}(x)=\tilde{E}\left(T^{-1}(z)\right)$ for all $z(t) \in T(D)$, and $T(D)=\left\{z \in \mathbb{R}^{n}:\|z(t)\|<r_{0}\right\}$ where $r_{0}$ represents a positive scalar.

In (27), $\zeta_{p}(z, t)$ represents the matched uncertainty associated with the primary control channels. The signal $\zeta_{p}(z, t)$ is assumed to be worst-case norm bounded by a known function $\bar{\zeta}_{p}(z, t)$. As before the actuator fault effectiveness matrix $W(t)$ must be estimated by some fault detection scheme (e.g. see [26, 30]). A similar analysis to that in Section 2.2 is considered here: it is assumed that the matrix $W(t)$ cannot be estimated perfectly and the estimated value $\widehat{W}(t)=$ $\operatorname{diag}\left(\widehat{W}_{1}(t), \widehat{W}_{2}(t)\right)$ satisfies the relationship in (7).

Let $\Delta(t)=\operatorname{diag}\left(\Delta_{1}(t), \Delta_{2}(t)\right)$ where $\Delta_{1}(t) \in \mathbb{R}^{l \times l}$ and $\Delta_{2}(t) \in \mathbb{R}^{(m-l) \times(m-l)}$ are unknown diagonal matrices such that

$$
\max \left(\left\|\Delta_{1}(t)\right\|,\left\|\Delta_{2}(t)\right\|\right) \leq \delta_{\max }<1
$$

Specifically, it follows from (7) that

$$
\begin{aligned}
& W_{1}(t)=\left(I_{l}-\Delta_{1}(t)\right) \widehat{W}_{1}(t) \\
& W_{2}(t)=\left(I_{m-l}-\Delta_{2}(t)\right) \widehat{W}_{2}(t)
\end{aligned}
$$

Assumption 2.1

Assume there already exists a primary control law

$$
u_{0}(t):=K(z), \quad K(0)=0
$$

that ensures the fault free closed-loop system

$$
\dot{z}(t)=F(z, t)+E_{0}(z) K(z):=F_{c}(z, t)
$$

where $E_{0}(z)$ is defined in (27), is asymptomatically stable for all $z(0) \in T(D)$. Equation (31) represents a nominal system with 'ideal performance'. 
This assumption formalizes the earlier statement that 'the system is over actuated' since asymptotic stability of (31) and hence (1) when $W(t)=I_{m}$ can be ensured using only the first $l$ control inputs, i.e. a control input to the healthy actuators of the form

$$
u(t)=\left[\begin{array}{c}
K(z) \\
0
\end{array}\right]
$$

stabilizes the system in (1).

\subsubsection{Control allocation component}

A control allocation scheme will now be created based on the existing controller in (30) for the primary surfaces. Define the physical control signals to be sent to all the actuators as

$$
u(t)=N(z) v(t)
$$

where $v(t) \in \mathbb{R}^{l}$ is the virtual control and $N(z) \in \mathbb{R}^{m \times l}$ is the control allocation matrix given by

$$
N(z)=\left[\begin{array}{c}
I_{l} \\
N_{2}(z)\left(I_{l}-\widehat{W}_{1}(t)\right)
\end{array}\right]
$$

where the component

$$
N_{2}(z)=E_{22}(z)^{T}\left(E_{22}(z) \widehat{W}_{2}(t) E_{22}(z)^{T}\right)^{-1}
$$

\section{Remark 2.5}

Note that $E_{22}(z) \in \mathbb{R}^{l \times(m-l)}$ and hence a necessary condition for $E_{22}(z) \widehat{W}_{2} E_{22}(z)^{T}$ in (35) to be nonsingular is that $m-l \geq l$. Furthermore at most $m-2 l$ of the diagonal elements of $\widehat{W}_{2}$ are allowed to be zero (i.e. failed). Although it is not straightforward to judge if $N(z)$ is full rank for a nonlinear system, in many real engineering systems such condition has physical meaning and the system to be controlled is designed to possess these properties at least locally to the operating conditions. (In the example discussed later in the paper, the rank condition of $N(z)$ is easy to verify.)

Consequently define the allowable fault/failure set as

$$
\mathcal{W}_{\epsilon}=\left\{\widehat{W}=\operatorname{diag}\left(\widehat{W}_{1}, \widehat{W}_{2}\right): \operatorname{det}\left(E_{22}(z) \widehat{W}_{2} E_{22}(z)^{T}\right) \neq 0 \quad \forall z \in T(D)\right\}
$$

Substituting (33) and (29) into (27) yields

$$
\begin{aligned}
\dot{z}(t)= & F(z, t)+\left[\begin{array}{c}
E_{12}(z)\left(I_{m-l}-\Delta_{2}\right) \widehat{W}_{2} N_{2}(z)\left(I_{l}-\widehat{W}_{1}\right) \\
E_{21}(z)\left(I_{l}-\Delta_{1}\right) \widehat{W}_{1}+E_{21}(z) E_{22}(z)\left(I_{m-l}-\Delta_{2}\right) \widehat{W}_{2} N_{2}(z)\left(I_{l}-\widehat{W}_{1}\right)
\end{array}\right] v(t) \\
& +\left[\begin{array}{c}
0 \\
E_{21}(z)
\end{array}\right] \delta_{p}(z, t)
\end{aligned}
$$

Using the definition of $N_{2}(z)$ in equation (35)

$$
\begin{aligned}
E_{21}(z) E_{22}(z) \widehat{W}_{2} N_{2}(z) & =E_{21}(z) E_{22}(z) \widehat{W}_{2} E_{22}(z)^{T}\left(E_{22}(z) \widehat{W}_{2} E_{22}(z)^{T}\right)^{-1} \\
& =E_{21}(z)
\end{aligned}
$$

Substituting (35) and (38) into (37) yields

$$
\begin{aligned}
\dot{z}(t)= & F(z, t)+\left[\begin{array}{c}
E_{12}(z)\left(I_{m-l}-\Delta_{2}\right) \widehat{W}_{2} E_{22}(z)^{T}\left(E_{22}(z) \widehat{W}_{2}(t) E_{22}(z)^{T}\right)^{-1}\left(I_{l}-\widehat{W}_{1}\right) \\
E_{21}(z)\left(I_{l}-\Delta_{1}\right) \widehat{W}_{1}+E_{21}(z)\left(I_{l}-\widehat{W}_{1}\right)
\end{array}\right] v(t) \\
& +\left[\begin{array}{c}
0 \\
E_{21}(z)
\end{array}\right] \delta_{p}(z, t) \\
= & F(z, t)+\underbrace{\left[\begin{array}{c}
E_{12}(z)\left(I_{m-l}-\Delta_{2}\right) E_{22}(z)^{\dagger}\left(I_{l}-\widehat{W}_{1}\right) \\
E_{21}(z)\left(I_{l}-\Delta_{1} \widehat{W}_{1}\right)
\end{array}\right]}_{E_{w}(z)} v(t)+\left[\begin{array}{c}
0 \\
E_{21}(z)
\end{array}\right] \delta_{p}(z, t)
\end{aligned}
$$


where

$$
E_{22}(z)^{\dagger}=\widehat{W}_{2} E_{22}(z)^{T}\left(E_{22}(z) \widehat{W}_{2}(t) E_{22}(z)^{T}\right)^{-1}
$$

represents a weighted right pseudo inverse of $E_{22}(z)$.

For a given $z$, since $\widehat{W}_{2}$ is diagonal, the pseudo inverse $E_{22}(z)^{\dagger}$ is norm bounded for all $z \in T(D)$ (see $[47,48]$ for details). Thus

$$
\left\|\left(I_{m-l}-\Delta_{2}\right) E_{22}(z)^{\dagger}\left(I_{l}-\widehat{W}_{1}\right)\left(I_{l}-\Delta_{1} \widehat{W}_{1}\right)^{-1}\right\| \leq \gamma_{0} \quad \forall z \in T(D), \widehat{W} \in \mathcal{W}_{\epsilon}
$$

for some positive scalar $\gamma_{0}$ since $\left\|\widehat{W}_{1}\right\|<1$.

In a fault-free situation with perfect fault estimation ( $\Delta=0$ and $\widehat{W}=I$ ), equation (39) becomes

$$
\dot{z}(t)=F(z, t)+E_{0}(z)\left(v(t)+\zeta_{p}(z, t)\right)
$$

where $E_{0}(z)$ is defined in (27). If the uncertainty $\zeta_{p}(z, t)=0$ and the virtual control $v(t)$ in (33) is chosen as $v=u_{0}$ where $u_{0}$ in turn is defined in (30), 'ideal' nominal performance is achieved. Also, in a fault-free situation with perfect estimation of $W(t)$ (i.e. $\Delta(t)=0$ and $\widehat{W}_{1}=I_{l}$ )

$$
N(z)=\left[\begin{array}{l}
I \\
0
\end{array}\right] \Rightarrow u=N(z) v=\left[\begin{array}{c}
u_{0} \\
0
\end{array}\right]
$$

Thus it can be seen from (43) that only the primary actuators are used in the fault-free situation.

\subsubsection{Switching function}

Choose the (integral) sliding surface as

$$
S=\left\{z \in \mathbb{R}^{n}: \sigma(z, t)=0\right\}
$$

where the switching function

$$
\sigma(z, t):=G z(t)-G z\left(t_{0}\right)-G \int_{0}^{t}\left(F(z, \tau)+E_{o}(z) K(z)\right) d \tau
$$

where $E_{o}(z)$ is defined in (27). Unlike Design 1 where the scaling matrix $G(x)$ is varying, here $G \in \mathbb{R}^{l \times n}$ is fixed and as a consequence the design of the equivalent injection signal can be simplified. The choice of design freedom $G$ employed here is

$$
G:=\left[\begin{array}{ll}
0 & I_{l}
\end{array}\right]
$$

By selecting $G$ as in (46), it follows

$$
G E_{o}(z)=E_{21}(z) \text { and } G E_{w}(z)=E_{21}(z)\left(I_{l}-\Delta_{1} \widehat{W}_{1}\right)
$$

Since $\Delta_{1}$ and $\widehat{W}_{1}$ are diagonal, $\left\|\widehat{W}_{1}\right\| \leq 1$ and $\left\|\Delta_{1}\right\|<\delta_{\max }<1$, it follows $\operatorname{det}\left(I_{l}-\Delta_{1} \widehat{W}_{1}\right) \neq 0$. Then using the fact that $E_{21}(z)$ is nonsingular, $G E_{w}(z)$ is nonsingular.

Using (39) and (45), the derivative of the switching function is

$$
\dot{\sigma}=G E_{w}(z) v-G E_{o}(z)\left(K(z)-\zeta_{p}(z, t)\right)
$$

where $E_{w}(z)$ is defined in (39). During sliding $\sigma=\dot{\sigma}=0$, then exploiting the fact the gain $G E_{w}(z) \in \mathbb{R}^{l \times l}$ is nonsingular, the equivalent control signal [1] required to maintain sliding is

$$
\begin{aligned}
v_{e q} & =\left(G E_{w}(z)\right)^{-1}\left(G E_{o}(z) K(z)-G E_{o}(z) \zeta_{p}(z, t)\right) \\
& =\left(I_{l}-\Delta_{1} \widehat{W}_{1}\right)^{-1}\left(K(z)-\zeta_{p}(z, t)\right)
\end{aligned}
$$


Substituting (49) into (39) yields

$$
\begin{aligned}
\dot{z}= & F(z, t)+\left[\begin{array}{c}
E_{12}(z)\left(I_{m-l}-\Delta_{2}\right) E_{22}(z)^{\dagger}\left(I_{l}-\widehat{W}_{1}\right) \\
E_{21}(z)\left(I_{l}-\Delta_{1} \widehat{W}_{1}\right)
\end{array}\right]\left(I_{l}-\Delta_{1} \widehat{W}_{1}\right)^{-1}\left(K(z)-\zeta_{p}\right)+E_{0}(z) \zeta_{p} \\
= & F(z, t)+\left[\begin{array}{c}
E_{12}(z)\left(I_{m-l}-\Delta_{2}\right) E_{22}(z)^{\dagger}\left(I_{l}-\widehat{W}_{1}\right)\left(I_{l}-\Delta_{1} \widehat{W}_{1}\right)^{-1} \\
0
\end{array}\right]\left(K(z)-\zeta_{p}\right)+E_{0}(z) \zeta_{p} \\
& +\left[\begin{array}{c}
0 \\
E_{21}(z)
\end{array}\right]\left(K(z)-\zeta_{p}\right) \\
= & F_{c}(z, t)+\left[\begin{array}{c}
E_{12}(z)\left(I_{m-l}-\Delta_{2}\right) E_{22}(z)^{\dagger}\left(I_{l}-\widehat{W}_{1}\right)\left(I_{l}-\Delta_{1} \widehat{W}_{1}\right)^{-1} \\
0
\end{array}\right]\left(K(z)-\zeta_{p}\right)
\end{aligned}
$$

where $F_{c}(z, t)$ is defined in (31). Note when the primary actuators are all healthy with perfect fault estimation $\widehat{W}_{1}=I_{l}$ and $\zeta_{p}(z, t)=0,(50)$ reduces to $\dot{z}=F_{c}(z, t)$ i.e. nominal closed-loop performance.

\subsubsection{Closed-loop stability}

Define $\phi=\left(I_{m-l}-\Delta_{2}\right) E_{22}(z)^{\dagger}\left(I_{l}-\widehat{W}_{1}\right)\left(I_{l}-\Delta_{1} \widehat{W}_{1}\right)^{-1}\left(K(z)-\zeta_{p}(z, t)\right)$ and consider (50) as an interconnection of two operators $\Sigma_{1}: \phi \mapsto \xi$ and $\Sigma_{2}: \xi \mapsto \phi$ given by

$$
\begin{aligned}
& \Sigma_{1}:\left\{\begin{array}{l}
\dot{z}=F_{c}(z, t)+\underbrace{\left[\begin{array}{c}
E_{12}(z) \\
0
\end{array}\right]}_{E_{1}(z)} \phi \\
\xi=z
\end{array}\right. \\
& \Sigma_{2}: \phi=\left(I_{m-l}-\Delta_{2}\right) E_{22}(\xi)^{\dagger}\left(I_{l}-W_{1}\right)\left(I_{l}-\Delta_{1} \widehat{W}_{1}\right)^{-1}\left(K(\xi)-\zeta_{p}(\xi, t)\right)
\end{aligned}
$$

Theorem 2.1

Let $\gamma_{1}$ be a positive scalar and assume there is a continuously differentiable, positive semidefinite function $\mathcal{V}(z)$ that satisfies the inequality

$$
\frac{\partial \mathcal{V}}{\partial z} F_{c}(z)+\frac{1}{2 \gamma_{1}^{2}} \frac{\partial \mathcal{V}}{\partial z} E_{1}(z) E_{1}(z)^{T}\left(\frac{\partial \mathcal{V}}{\partial z}\right)^{T}+\frac{1}{2} K(z)^{T} K(z) \leq 0
$$

for all $z \in T(D)$. Then if $\gamma_{1} \gamma_{0}<1$, where $\gamma_{0}$ is defined in (41), the sliding motion in (50) is asymptotically stable.

\section{Proof}

Using the assumption that $K(0)=0$ and the fact that $f(0, t)=0$, it is straightforward to verify $F(0, t)=F_{c}(0, t)=0$. Inequality (53) is known as the Hamilton-Jacobi inequality associated with the operator $\Sigma_{1}$ (see Theorem 5.5 in [58]). Then, for each $z(0) \in T(D)$, the operator $\Sigma_{1}$ is finitegain $\mathcal{L}_{2}$ stable and its $\mathcal{L}_{2}$ gain is less than or equal to $\gamma_{1}$. From (41), the $\mathcal{L}_{2}$ gain associated with $\Sigma_{2}$ is less than or equal to $\gamma_{0}$ and therefore a small gain argument guarantees the asymptomatic stability of (50) when $\gamma_{1} \gamma_{0}<1$.

\section{Remark 2.6}

Although solving (53) is not straightforward [58], numerical attempts (e.g. [59, 60]) have been developed in the literature. The work in [59] tries to solve Hamilton-Jacobi inequality using the Converse Lyapunov theorem. As argued in [60], if there exists a vector field $\beta(z)$ such that

$$
\beta(z)^{T} \psi(z)^{\dagger} \beta(z)-F_{c}(z)^{T} \psi(z)^{\dagger} F_{c}(z)+K(z)^{T} K(z) \leq 0
$$

where

$$
\psi(z)=\frac{1}{\gamma_{1}^{2}} E(z) E(z)^{T}
$$


then the solution

$$
\mathcal{V}(z)=\int_{0}^{z}\left(-F_{c}(\tau) \pm \beta(\tau)\right)^{T} \psi(\tau)^{\dagger} d \tau
$$

will satisfy (53) for $z \in T(D)$.

\subsubsection{Sliding mode control law}

This section describes the appropriate choice of the virtual control law to maintain sliding.

Proposition 2.1

Consider the virtual control based on the integral sliding mode control law

$$
v(t)=u_{0}(t)+v_{n}(t)
$$

where $u_{0}(t)$ is from (30) and represents the baseline controller which ensures ideal performance in the fault free situation, and the retro-fitted component

$$
v_{n}(t):=-\rho(t, z) \frac{E_{21}(z)^{T} \sigma(t, z)}{\left\|E_{21}(z)^{T} \sigma(t, z)\right\|}
$$

where $\rho(t, z)$ is the modulation gain. If the modulation gain is selected as

$$
\rho(t, z) \geq \frac{\left(\delta_{\max }\left\|u_{0}\right\|+\bar{\zeta}_{p}(z, t)\right)\left\|E_{21}(z)\right\|+\eta_{0}}{\left(1-\delta_{\max }\right)\left\|E_{21}(z)\right\|}
$$

sliding will be maintained in the presence of the actuator fault/failure represented by $W(t) \in \mathcal{W}_{\epsilon}$.

Proof

Using the fact in (47) and substituting (58) into (48) yields

$$
\begin{aligned}
\dot{\sigma} & =E_{21}(z)\left(I_{l}-\Delta_{1} \widehat{W}_{1}\right)\left(K(z)-\rho(t, z) \frac{E_{21}(z)^{T} \sigma(t, z)}{\left\|E_{21}(z)^{T} \sigma(t, z)\right\|}\right)-E_{21}(z)\left(K(z)-\zeta_{p}(z, t)\right) \\
& =-E_{21}(z) \Delta_{1} \widehat{W}_{1}\left(K(z)-\rho(t, z) \frac{E_{21}(z)^{T} \sigma(t, z)}{\left\|E_{21}(z)^{T} \sigma(t, z)\right\|}\right)-E_{21}(z) \rho(t, z) \frac{E_{21}(z)^{T} \sigma(t, z)}{\left\|E_{21}(z)^{T} \sigma(t, z)\right\|}+E_{21}(z) \zeta_{p}(z, t)
\end{aligned}
$$

Clearly

$$
\begin{aligned}
\sigma^{T} \dot{\sigma} & \leq-\rho\left\|E_{21}(z)^{T} \sigma\right\|+\left\|E_{21}(z)^{T} \sigma\right\|\left\|\Delta_{1}\right\|\left\|u_{0}\right\|+\rho\left\|E_{21}(z)^{T} \sigma\right\|\left\|\Delta_{1}\right\|+\left\|E_{21}(z)^{T} \sigma\right\| \bar{\zeta}_{p}(z, t) \\
& \leq-\rho\left(1-\left\|\Delta_{1}\right\|\right)\left\|E_{21}(z)^{T} \sigma\right\|+\left\|E_{21}(z)^{T} \sigma\right\|\left\|\Delta_{1}\right\|\left\|u_{0}\right\|+\left\|E_{21}(z)^{T} \sigma\right\| \bar{\zeta}_{p}(z, t) \\
& \leq-\rho\left(1-\delta_{\max }\right)\left\|E_{21}(z)\right\|\|\sigma\|+\delta_{\max }\left\|E_{21}(z)\right\|\|\sigma\|\left\|u_{0}\right\|+\left\|E_{21}(z)^{T}\right\|\|\sigma\| \bar{\zeta}_{p}(z, t)
\end{aligned}
$$

By choosing the modulation gain as proposed in (59), the inequality in (61) can be written as $\sigma^{T} \dot{\sigma} \leq-\eta_{0}\|\sigma\|$ which represents the standard reachability condition [1].

Substituting (57) and (58) into (33), the physical sliding mode control law yields the expression

$$
u(t)=\left[\begin{array}{c}
I_{l} \\
N_{2}(z)\left(I_{l}-\widehat{W}_{1}(t)\right)
\end{array}\right]\left(K(z)-\rho(t, z) \frac{E_{21}(z)^{T} \sigma(t, z)}{\left\|E_{21}(z)^{T} \sigma(t, z)\right\|}\right)
$$

Remark 2.7

Equation (62) clearly shows the architecture of FTC scheme: the original control $u_{0}=K(z)$ is augmented with a nonlinear term to induce sliding and this control signal is distributed to the physical actuators through the control allocation matrix $N_{2}$.

\section{Remark 2.8}

It is well known that the discontinuous control law in (62) will generate chattering. In this paper, in the case study, the discontinuous control signal is approximated by a continuous signal by approximating the unit vector with a sigmoid function $[1,2]$. An alternative way of developing a continuous controller straightforwardly is to use higher order sliding modes (e.g. the work in $[44,61]$ proposes a continuous integral sliding mode fault tolerant control allocation scheme). 


\section{DESIGN EXAMPLE}

This approach will now be demonstrated using a nonlinear model of the longitudinal motion of a rigid aircraft [54, 62].

\subsection{Longitudinal aircraft model}

Consider

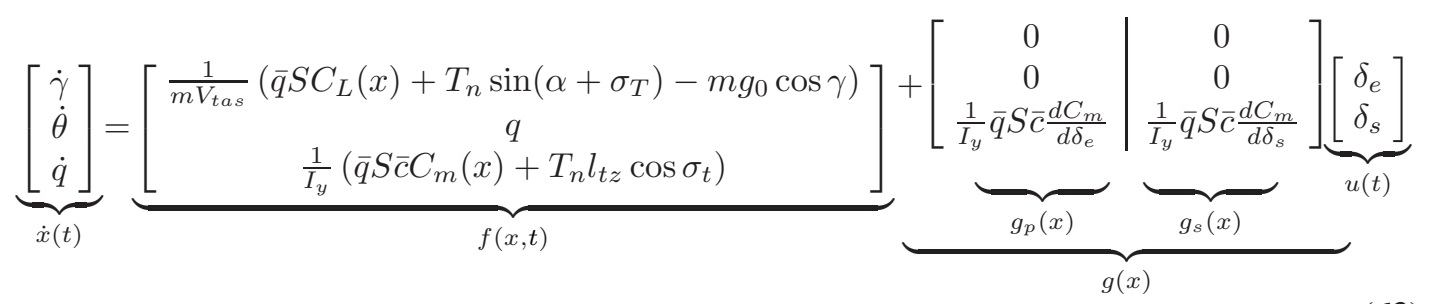

where the system states $\gamma, \theta, q$ denote flight path angle (FPA), pitch angle and pitch rate, respectively. The system inputs $\delta_{e}, \delta_{s}$ represent the elevator and horizontal stabilizer deflections respectively. The variables $V_{\text {tas }}, \alpha, \bar{q}, C_{L}(x), C_{m}(x)$ in (63) denote true air speed, angle of attack, dynamic pressure, the component of the lift force coefficient and the component of the pitch moment coefficient respectively. It is assumed that an auto throttle loop exists which maintains $V_{\text {tas }}$ at a constant value. The known parameters $m, g_{0}, I_{y}, T_{n}, l_{t z}, \sigma_{T}, S, \bar{c}$ represent mass, gravity, the body axis moment of inertia, total engine thrust, the distance from the engine centre line to the fuselage reference line, the engine inclination angle, the wing area and the wing mean aerodynamic chord respectively.

In (63), the terms $\frac{d C_{m}}{d \delta_{e}}$ and $\frac{d C_{m}}{d \delta_{s}}$ are typically available from online estimation or a lookup table. As in [63], the vector $g_{p}(x)$ is associated with the primary control surface (the elevator) and the vector $g_{s}(x)$ is associated with the secondary control surface (the stabilizer) which will be used when faults/failures occur on the primary control surface.

From (63), $f(0) \approx 0$ and it is easy to verify $g_{p}(x)$ and $g_{s}(x)$ are slow varying and smooth using the data extracted from [54]. In this example, a coordinate transformation is necessary. Consequently rewrite (63) taking into consideration the effect of actuator faults as

$$
\dot{z}(t)=f(z, t)+\underbrace{\left[\begin{array}{cc}
0 & 0 \\
g_{21}(z) & g_{21}(z) g_{22}(z)
\end{array}\right]}_{g(z)} W u(t)
$$

where $z=x, g_{21}(z)=\frac{1}{I_{y}} \bar{q} S \bar{c} \frac{d C_{m}}{d \delta_{e}}$ and $g_{22}(z)=\frac{1}{I_{y}} \bar{q} S \bar{c} \frac{d C_{m}}{d \delta_{s}} / g_{21}(z)$. For this particular system, $W(t):=\operatorname{diag}\left(w_{e}(t), w_{s}(t)\right)$ where the time varying scalars $w_{e}(t)$ and $w_{s}(t)$ represent the effectiveness of the elevator (the primary actuator) and the stabilizer (the secondary actuator) respectively. It is assumed that $w_{e}(t)$ and $w_{s}(t)$ cannot be perfectly estimated. The estimated effectiveness values of the actuators are given by $\widehat{W}(t):=\operatorname{diag}\left(\hat{w}_{e}(t), \hat{w}_{s}(t)\right)$.

Clearly from (64), the system in (63) is formulated as (27) with $E_{12}(z)=0$. Consequently, the proposed procedure will now be applied to the longitudinal aircraft model.

\subsection{Design results}

In this example, the nominal inner-loop control law $\left(u_{0}(t)\right.$ in $\left.(30)\right)$ has been created by using the back-stepping procedure developed in [50,51]. Specifically

$$
u_{0}(t)=K(z)=\left(\frac{d C_{m}}{d \delta_{e}}\right)^{-1}\left(\frac{I_{y} \dot{q}_{d e s}-\bar{q} S \bar{c} C_{m}(x)-T_{n} l_{t z} \cos \sigma_{t}}{\bar{q} S \bar{c}}\right)
$$

where

$$
\dot{q}_{d e s}=-\left[\begin{array}{lll}
\kappa_{1} \kappa_{2} \kappa_{3} & \kappa_{2} \kappa_{3} & \kappa_{3}
\end{array}\right]\left[\begin{array}{c}
\gamma-\gamma_{\text {ref }} \\
\theta-\gamma_{r e f}-\alpha_{0} \\
q
\end{array}\right]
$$


and $\alpha_{0}$ is the angle of attack at a steady state condition [50]. In this example, the gains $\kappa_{1}, \kappa_{2}, \kappa_{3}$ are chosen as $\kappa_{1}=1, \kappa_{2}=0.6$ and $\kappa_{2}=3$ which satisfy

$$
\begin{aligned}
& \kappa_{1}>-1 \\
& \kappa_{2}>0 \\
& \kappa_{3}>\left\{\begin{array}{cl}
\kappa_{2} & \text { if } \kappa_{1} \leq 0 \\
\kappa_{2}\left(1+\kappa_{1}\right) & \text { if } \kappa_{1}>0
\end{array}\right.
\end{aligned}
$$

Notice that during the simulation, the altitude/FPA control and velocity are maintained using a separate outer-loop controller. Furthermore, an Instrument Landing System (ILS) is used to switch between an altitude/FPA command (in pilot commanded mode) and sideslip tracking (in automated landing mode) [63]. In this example, only the pilot command mode is active.

In this example, the control allocation matrix $N(z)$ is created from (34) as

$$
\begin{aligned}
N(z) & =\left[\begin{array}{c}
1 \\
g_{22}(z)^{T}\left(g_{22}(z) \hat{w}_{s}(t) g_{22}(z)^{T}\right)^{-1}\left(1-\hat{w}_{e}(t)\right)
\end{array}\right] \\
& =\left[\begin{array}{c}
1 \\
\left(1-\hat{w}_{e}(t)\right)\left(g_{22}(z) \hat{w}_{s}(t)\right)^{-1}
\end{array}\right]
\end{aligned}
$$

because all the terms are scalars.

The switching function $\sigma(t)$ is selected as

$$
\sigma(t):=G z(t)-G z\left(t_{0}\right)-G \int_{0}^{t}\left(f(z, t)+\left[\begin{array}{c}
0_{2 \times 1} \\
g_{21}(z)
\end{array}\right] K(z)\right) d \tau
$$

where $G=\left[\begin{array}{ll}0 & 1\end{array}\right]$ and $K(z)$ is defined in (65).

As defined in (62), the final online ISM/CA law is given by

$$
u(t)=N(z)\left(K(z)-\rho(t, z) \frac{g_{21}(z)^{T} \sigma(t, z)}{\left\|g_{21}(z)^{T} \sigma(t, z)\right\|+\delta}\right)
$$

where $\rho(t, z)$ is selected as 0.65 . For implementation purposes, a 'smooth' approximation of the discontinuous signal from (62) is used as shown in (70) where the smoothing factor $\delta$ is selected as 0.01 .

\subsection{Simulation results}

In this section, the proposed (retro-fitted) scheme has been tested on the nonlinear RECOVER benchmark model which represents a high fidelity model of a large transport aircraft (B747) and has been used in the GARTEUR AG16 programme for the study of FTC schemes [41]. All the simulations have been conducted at a trim altitude of $2000 \mathrm{~m}$, a mass of 263 tons, $25 \%$ centre gravity, a speed of $92.6 \mathrm{~m} / \mathrm{s}$ and flap settings of 20deg. During the simulation, an altitude change manoeuvre has been commanded and an elevator fault is assumed to occur from 100sec onwards. In this paper, two simulations are presented where a loss of effectiveness (LOE) fault scenario and an elevator lock-in-place failure scenario are considered. In the LOE fault scenario, it is assumed that there exists a time varying estimation error in $w_{e}$ associated with the elevator. In the lock-inplace failure scenario, the elevator is assumed to be stuck (i.e. $w_{e}=0$ ) at an offset position in the presence of an imperfect fault estimation $\hat{w}_{e}=0.2$. To demonstrate the efficacy of the scheme, the system performance in the fault free case and in the case when faults/failures occur on the elevator in the presence of imperfect knowledge of elevator effectiveness estimation will be compared. The simulation results associated with the loss of effectiveness fault scenario are shown in Figs. 3-6. Fig. 3 shows the trajectories of the aircraft states. It can be seen from Fig. 3 that the proposed scheme can achieve good fault free (close to nominal) performance despite the elevator fault and a time varying error in the estimate of the elevator effectiveness level. As shown in Fig. 4, the altitude change manoeuvre is assumed to start at $50 \mathrm{sec}$. Clearly, the fault free trajectories of the 

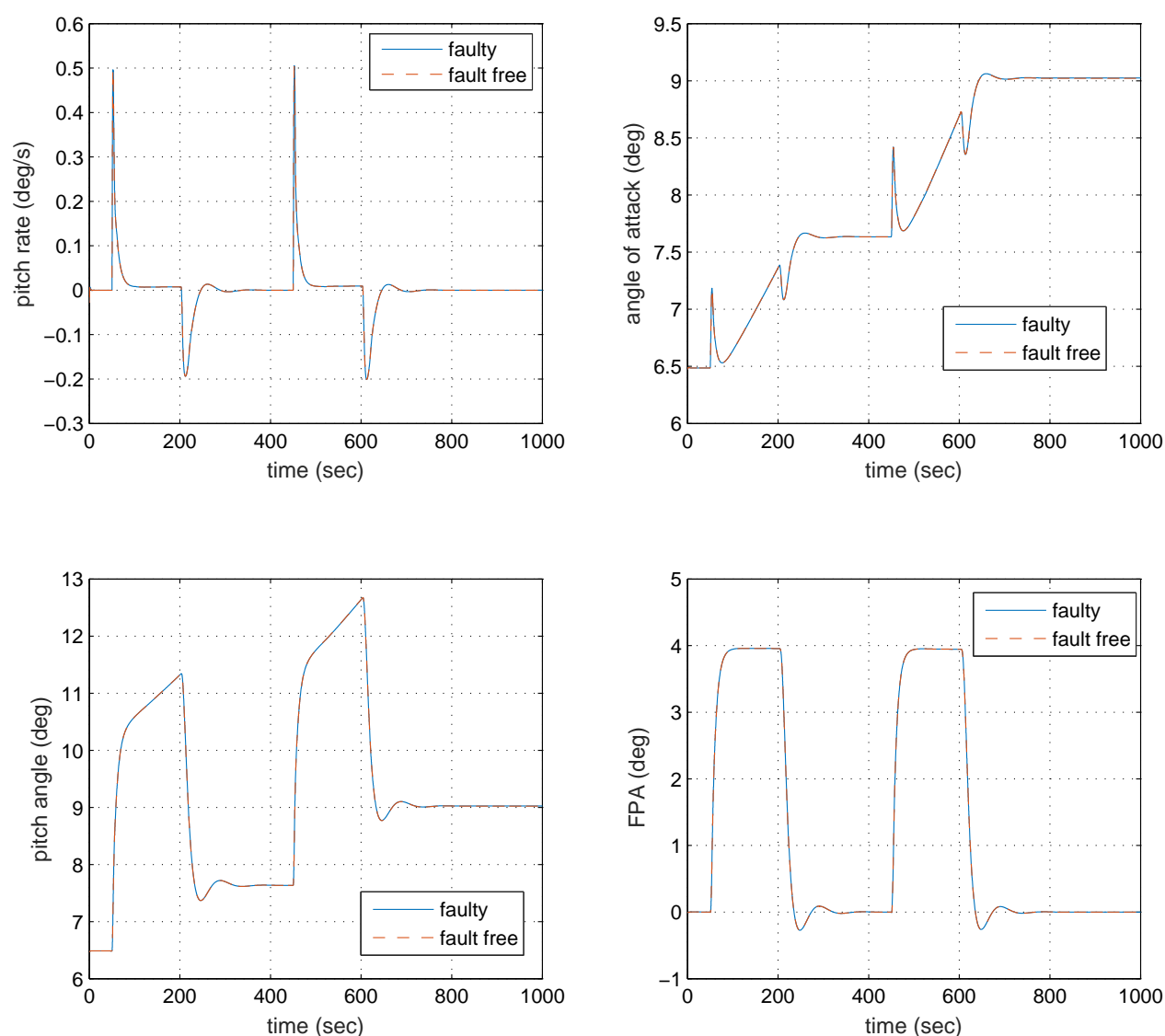

Figure 3. The trajectories of aircraft states (Loss of effectiveness)

altitude can be retained in the presence of the fault. In this paper, the elevator fault (i.e. a reduction of elevator effectiveness level) occurred at 100sec. It can be seen from Fig. 5, both the elevator and the stabilizer try to compensate after the occurrence of the fault. As shown in Fig. 6, the switching functions are maintained close to zero for both the fault free and faulty cases. The effect of imperfect estimation of the elevator effectiveness level is also shown in Fig. 6. In Fig. 6, the blue line and the red line represent the actual effectiveness level $w_{e}$ and its estimated value $\hat{w}_{e}$ respectively. Clearly, the estimated effectiveness level $\hat{w}_{e}$ contains a time varying estimation error. Figs. 3-6 demonstrate the efficacy of the online ISM/CA scheme.

Figs 7-10 show a comparison between the fault free case and the situation when the elevator lock-in-place failure occurs at an offset position from 100sec onwards. In this case, the actual effectiveness level $w_{e}=0$ (because of the actuator failure), but it is assumed that $\hat{w}_{e}=0.2$ due to an imperfect estimation of $w_{e}$. The state trajectories of the aircraft are shown in Fig. 7 which demonstrate that fault free performance can be retained despite the actuator failure and the imperfect estimation error. From Fig. 9 it is clear that once the elevator lock-in-place failure occurs at 100sec, the stabilizer becomes active due to a reallocation of the control signals. The switching functions, as shown in Fig. 10, are close to zero for both the fault free and failure cases. The actual elevator effectiveness level (blue line) and its estimate (red line) are shown in Fig. 10.

\section{CONCLUSION}

This paper has proposed online ISM/CA schemes for a class of overactuated nonlinear affine systems with the objective of retaining fault-free (nominal) performance in the face of actuator faults 

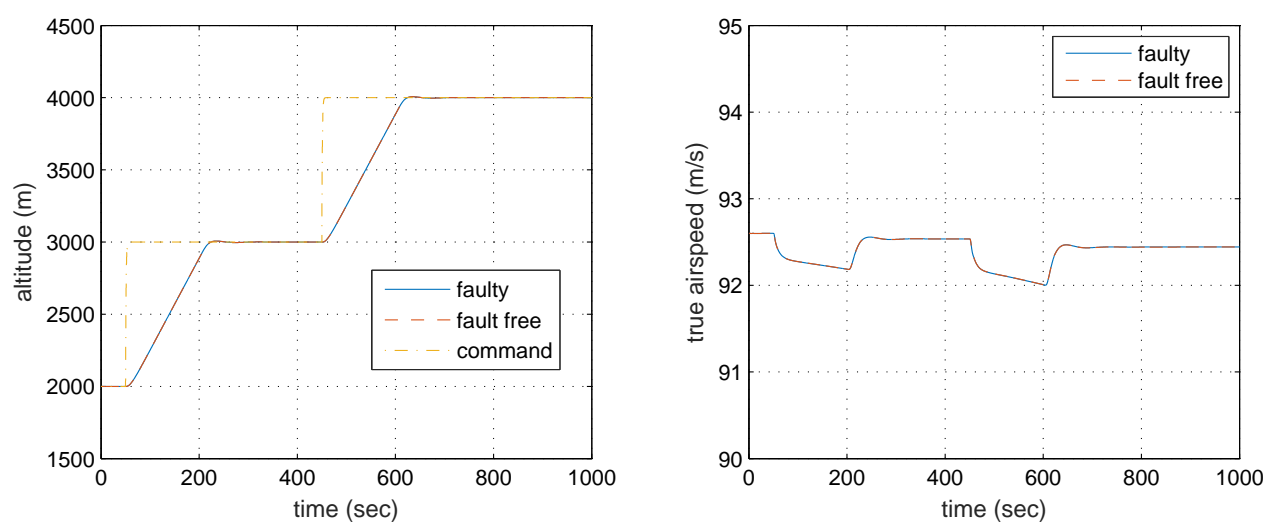

Figure 4. The trajectories of aircraft altitude and true airspeed (Loss of effectiveness)
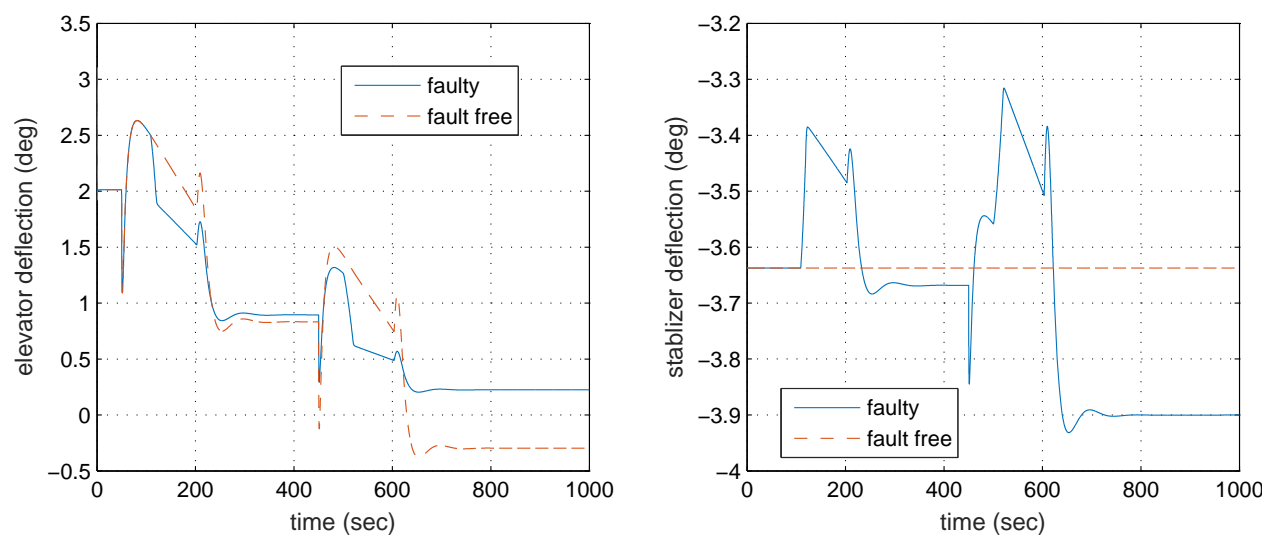

Figure 5. Control surface deflections (Loss of effectiveness)
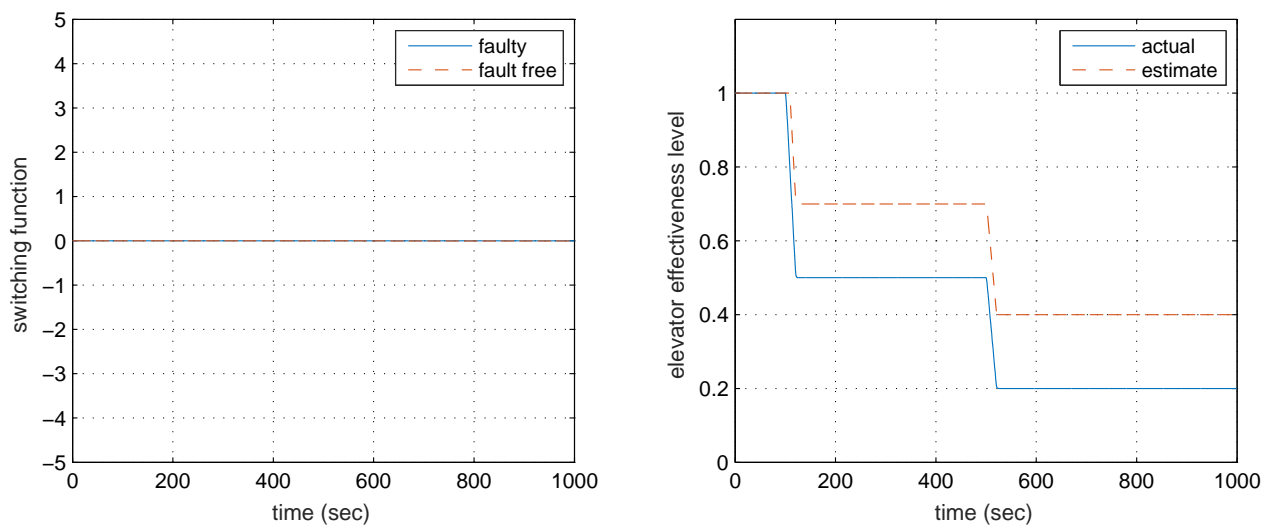

Figure 6. Switching function $\sigma(\cdot)$ and elevator effectiveness level $w_{e}$ (Loss of effectiveness)

and imperfect estimation of the actuator effectiveness levels. The paper extends earlier work based on linear plant models to a wider class of nonlinear systems. The advantage of the proposed schemes is that they can be integrated with existing baseline controllers thanks to the special structure of the ISM formulation. One of the proposed schemes has been applied to a high fidelity nonlinear aircraft simulation model. The simulation results show the efficacy of the scheme. 

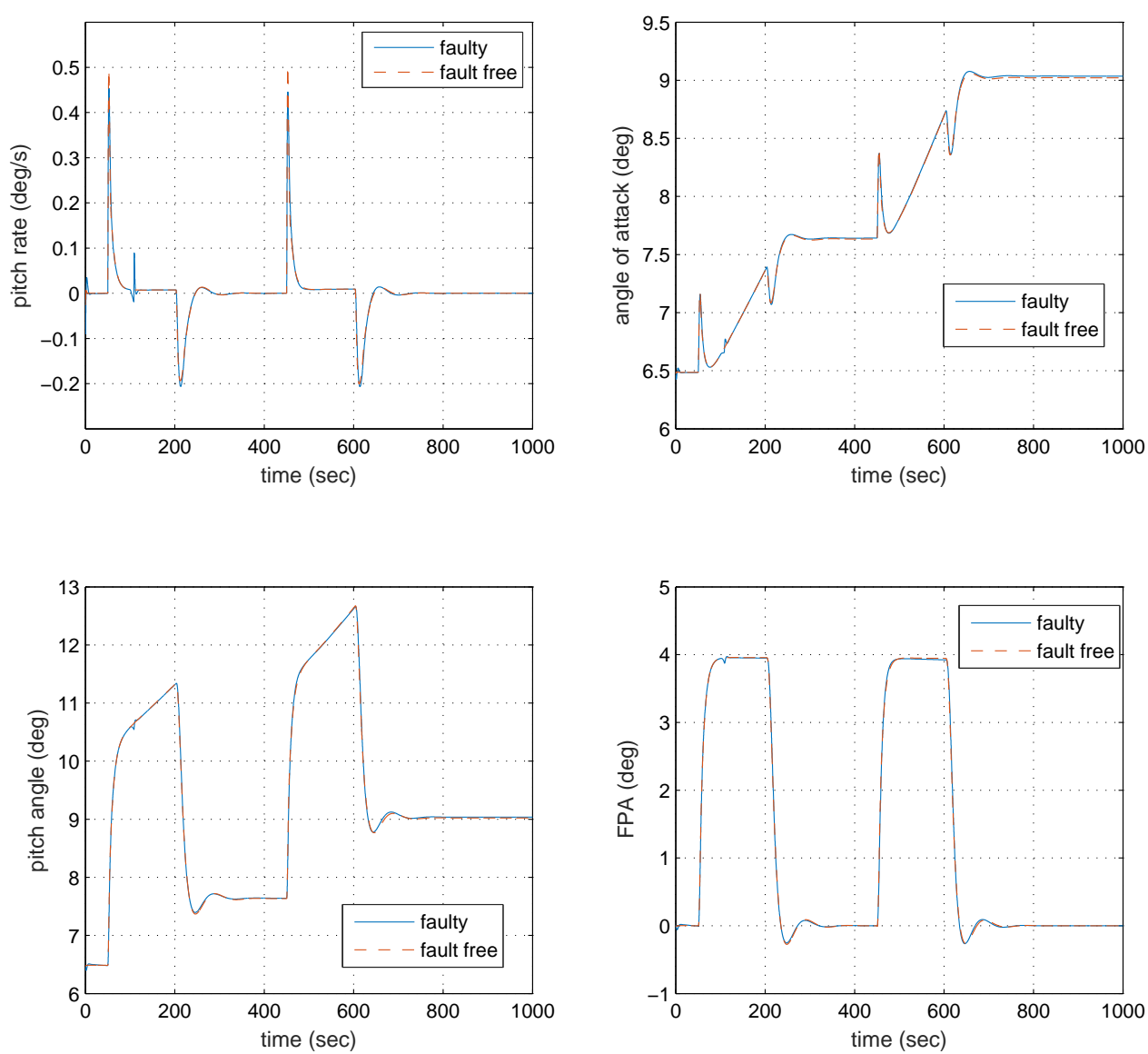

Figure 7. The trajectories of aircraft states (lock-in-place)
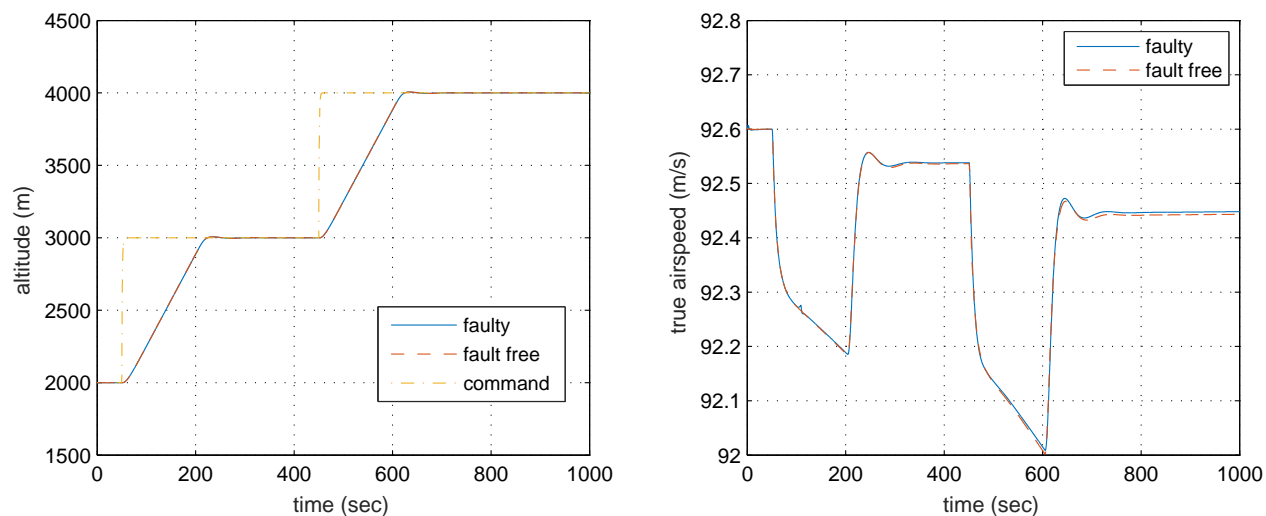

Figure 8. The trajectories of aircraft altitude and true airspeed (lock-in-place)

\section{REFERENCES}

1. Utkin V. Sliding modes in control and optimization. Springer: Heidelberg, 1992.

2. Edwards C, Spurgeon SK. Sliding Mode Control: Theory and Applications. London, U.K.: Taylor \& Francis, 1998.

3. Shtessel Y, Edwards C, Fridman L, Levant A. Sliding mode control and observation. Springer: Basel, 2013.

4. Matthews G, DeCarlo R. Decentralized tracking for a class of interconnected nonlinear systems using variable structure control. Automatica 1988; 24:187-193. 

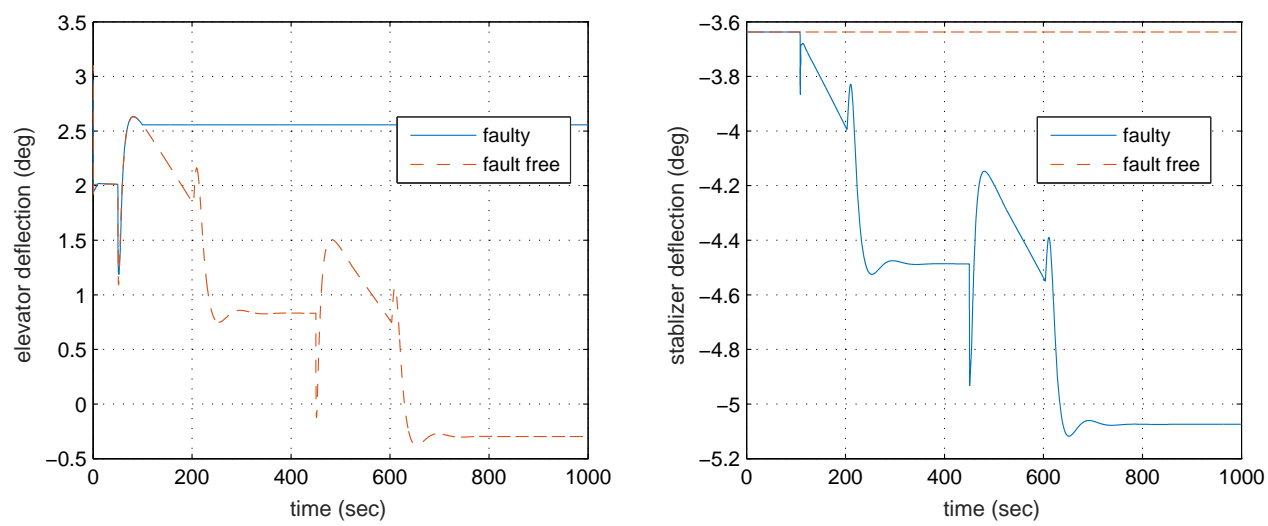

Figure 9. Control surface deflections (lock-in-place)
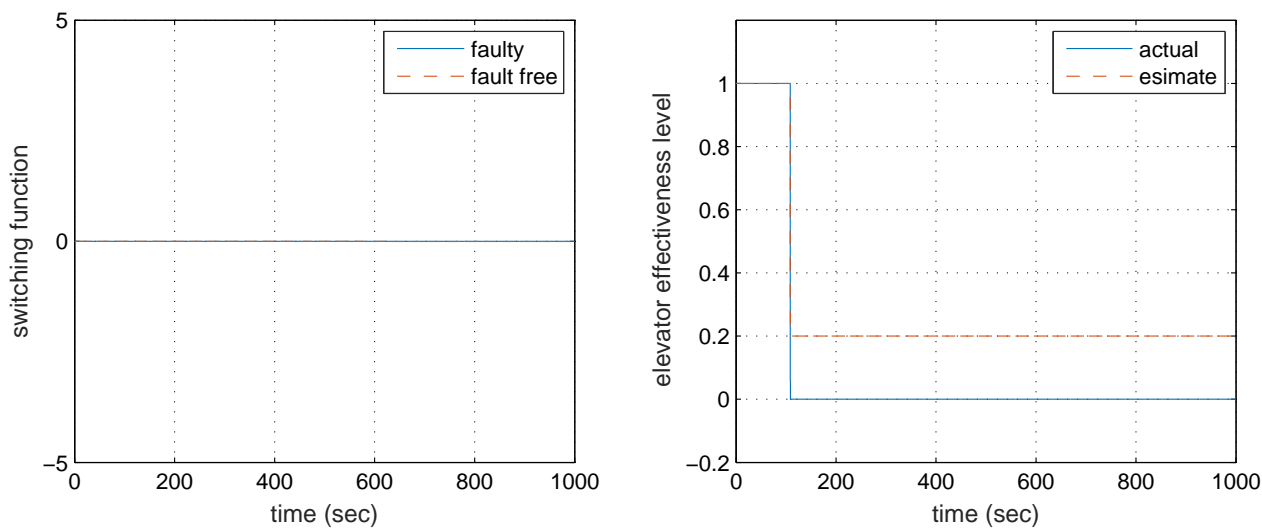

Figure 10. Switching function $\sigma(\cdot)$ and elevator effectiveness level $w_{e}$ (lock-in-place)

5. Utkin V, Shi J. Integral sliding mode in systems operating under uncertainty conditions. Proceedings of the IEEE $C D C, 1996 ; 4591-4596$.

6. Castanos F, Fridman L. Analysis and design of integral sliding manifolds for systems with unmatched perturbations. IEEE Trans. Autom. Control 2006; 51:2006.

7. Bejarano F, Fridman L, Poznyak A. Output integral sliding mode control based on algebraic hierarchical observer. Int. J. Control 2007; 2007:443-453.

8. Hamayun M, Edwards C, Alwi H. Design and analysis of an integral sliding mode fault tolerant control scheme. IEEE Trans. Autom. Control 2012; 57:1783-1789.

9. Fridman L, Poznyak A, Rodriguez FB. Robust Output LQ Optimal Control via Integral Sliding Modes. Birkhuser Base, 2014.

10. Rubagotti M, Estrada A, Castanos F, Ferrara A, Fridman L. Integral sliding mode control for nonlinear systems with matched and unmatched perturbations. IEEE Trans. Autom. Control 2011; 56:2699-2704.

11. Liu JS, Yuan K. On tracking control for affine nonlinear systems by sliding mode. Syst. Control Lett 1989; 13:439 $-443$.

12. Chen YC, Chang S. Output tracking design of affine nonlinear plant via variable structure system. IEEE Trans. Autom. Control 1992; 37:1823-1828.

13. Yan XG, Spurgeon SK, Edwards C. Memoryless static output feedback sliding mode control for nonlinear systems with delayed disturbances. IEEE Trans. Autom. Control 2014; 59:1906-1912.

14. Yan XG, Spurgeon SK, Zhu Q, Zhang Q. Memoryless variable structure control for affine nonlinear systems using only output information. Int. J. Robust Nonlinear Control 2015; 25:3316-3329.

15. Bartolini G, Punta E, Zolezzi T. Multi-input sliding mode control of nonlinear uncertain affine systems. Int. J. Control 2011; 84:867-875.

16. Benosman M, Lum KY. Passive actuators' fault-tolerant control for affine nonlinear systems. IEEE Trans. Control Syst. Technol. 2010; 18:152-163.

17. Incremona GP, Cucuzzella M, Ferrara A. Second order sliding mode control for nonlinear affine systems with quantized uncertainty. Automatica 2017; 86:46 - 52.

18. Levant A. Universal single-input-single-output (SISO) sliding-mode controllers with finite-time convergence. IEEE Transactions on Automatic Control 2001; 46:1447-1451. 
19. Dinuzzo F, Ferrara A. Higher order sliding mode controllers with optimal reaching. IEEE Trans. Autom. Control 2009; 54:2126-2136.

20. Laghrouche S, Plestan F, Glumineau A. Higher order sliding mode control based on integral sliding mode. Automatica 2007; 43:531 - 537.

21. Levant A, Alelishvili L. Integral high-order sliding modes. IEEE Transactions on Automatic Control 2007; 52:1278-1282.

22. Estrada A, Fridman LM. Integral HOSM semiglobal controller for finite-time exact compensation of unmatched perturbations. IEEE Trans. Autom. Control 2010; 55:2645-2649.

23. Chakrabarty A, Dinh V, Buzzard GT, Zak SH, Rundell AE. Robust explicit nonlinear model predictive control with integral sliding mode. Proc. Amer. Contr. Conf., 2014; 2851-2856.

24. Errouissi R, Yang J, Chen WH, Al-Durra A. Robust nonlinear generalised predictive control for a class of uncertain nonlinear systems via an integral sliding mode approach. International Journal of Control 2016; 89:1698-1710.

25. Fan QY, Yang G. Adaptive actorcritic design-based integral sliding-mode control for partially unknown nonlinear systems with input disturbances. IEEE Trans Neural Networks Learn Syst 2016; 27:165-177.

26. Chen J, Patton RJ. Robust model-based fault diagnosis for dynamic systems. Kluwer academic publishers: Norwell, 1999.

27. Ding SX. Model-Based Fault Diagnosis Techniques. Springer, 2013.

28. Zhang Y, Jiang J. Bibliographical review on reconfigurable fault-tolerant control systems. Annual Reviews in Control 2008; 32:229 - 252 .

29. Blanke M, Kinnaert M, Lunze J, Staroswiecki M. Introduction to Diagnosis and Fault-Tolerant Control. Springer Berlin Heidelberg: Berlin, Heidelberg, 2016; 1-35.

30. Alwi H, Edwards C, Tan CP. Fault Detection and Fault-Tolerant Control Using Sliding Modes. Springer: London, 2011.

31. Durham W, Bordignon KA, Beck R. Aircraft Control Allocation. John Wiley \& Sons: Chichester, 2017.

32. Johansen TA, Fossen TI. Control allocation - a survey. Automatica 2013; 49:1087-1103.

33. Zhang Y, Suresh VS, Jiang B, Theilliol D. Reconfigurable control allocation against aircraft control effector failures. 16th IEEE International Conference on Control Applications, Singapore, 2007; 1197-1202.

34. Lombaerts TJJ, Looye GHN, Chu QP, Mulder JA. Design and simulation of fault tolerant flight control based on a physical approach. Aerosp. Sci. Technol. 2012; 23:151-171.

35. Sato M, Peaucelle D. Gain-scheduled output-feedback controllers using inexact scheduling parameters for continuous-time LPV systems. Automatica 2013; 49:1019-1025.

36. Vanek B, Edelmayer A, Szabo Z, Bokor J. Bridging the gap between theory and practice in LPV fault detection for flight control actuators. Control Eng. Pract. 2014; 31:171-182.

37. Rotondo D, Nejjari F, Puig V, Blesa J. Model reference FTC for LPV systems using virtual actuator and setmembership fault estimation. Int. J. Robust Nonlinear Control 2015; 25:753-760.

38. Hamayun MT, Edwards C, Alwi H. Fault tolerant control schemes using integral sliding modes. Springer: Cham, 2016.

39. Liang YW, Ting LW, Lin LG. Study of reliable control via an integral-type sliding mode control scheme. IEEE Trans. Ind. Electron. 2012; 59:3062-3068.

40. Liang YW, Chen CC, Liaw DC, Wei YT. Nonlinear reliable control with application to a vehicle antilock brake system. IEEE Trans. Ind. Inf. 2013; 9:2114-2123.

41. Edwards C, Lombaerts T, Smaili H. Fault tolerant flight control: A benchmark challenge. Springer, 2010.

42. Hamayun MT, Edwards C, Alwi H. A fault tolerant control allocation scheme with output integral sliding modes. Automatica 2013; 49(6):1830-1837.

43. Alwi H, Edwards C, Stroosma O, Mulder J, Hamayun M. Real-time implementation of an integral sliding mode fault tolerant control scheme for LPV plants. IEEE Trans. Ind. Electron. 2015; 62:3896-3905.

44. Chen L, Alwi H, Edwards C. Development and evaluation of an integral sliding mode fault-tolerant control scheme on the reconfigure benchmark. Int. J. Robust Nonlinear Control 2017; 0(0):1-27, doi:10.1002/rnc.3951.

45. Jiang B, Staroswiecki M, Cocquempot V. Fault estimation in nonlinear uncertain systems using robust/sliding mode observers. IEE Proceedings - Control Theory and Applications 2004; 151(1):29-37.

46. Fridman L, Shtessel Y, Edwards C, Yan XG. Higher-order sliding-mode observer for state estimation and input reconstruction in nonlinear systems. International Journal of Robust and Nonlinear Control 2008; 18:399-412.

47. Stewart GW. On scaled projections and pseudoinverses. Linear Algebra and Its Applications 1989; 112:189-193.

48. Alwi H, Edwards C. Fault tolerant control using sliding modes with on-line control allocation. Automatica 2008; 44:1859-1866.

49. Isidori A. Nonlinear control systems (3rd ed.). Berlin: Springer-Verlag, 1995.

50. Harkegard O, Glad ST. A backstepping design for flight path angle control. Proceedings of the IEEE CDC, 2000; $3570-3575$

51. Harkegard O. Backstepping and control allocation with application to flight control. PhD Thesis, Linkoping University 2003.

52. Merola A, Cosentino C, Colacino D, Amato F. Optimal control of uncertain nonlinear quadratic systems. Automatica 2017; 83:345 - 350 .

53. Isidori A, Astolfi A. Disturbance attenuation and $H_{\infty}$ control via measurement feedback in nonlinear systems. IEEE Trans. Autom. Control 1992; 37:1283-1293.

54. Hanke C, Nordwall D. The simulation of a jumbo jet transport aircraft. Volume II: Modelling data. Technical Report NASA-CR-114494, D6-30643-VOL-2, ARC-E-DAA-TN9543, NASA and The Boeing Company 1970.

55. Vidyasagar M. Nonlinear Systems Analysis (Second Edition). Prentice Hall: Upper Saddle River, NJ, USA, 1993.

56. Marino R. Adaptive observers for single output nonlinear systems. IEEE Trans. Autom. Control. 1990; 35:10541058 .

57. Marino R, Tomei P. Nonlinear Control Design. Englewood Cliff: Prentice Hall International, 1995.

58. Khalil HK. Nonlinear Systems Third Edition. Pearson Education International Inc., 2000. 
59. Imura JI, Sugie T, Yoshikawa T. A Hamilton-Jacobi inequality approach to the strict $H_{\infty}$ control problem of nonlinear systems. Automatica 1996; 32(4):645 - 650.

60. Aliyu MDS. An approach for solving the Hamilton-Jacobi-Isaacs equation (HJIE) in nonlinear $H_{\infty}$ control. Automatica 2003; 39:877 - 884.

61. Rios H, Kamal S, Fridman LM, Zolghadri A. Fault tolerant control allocation via continuous integral sliding-modes: A HOSM-Observer approach. Automatica 2015; 51:318 - 325.

62. Marcos A, Balas GJ. A Boeing 747-100/200 aircraft fault tolerant and diagnostic benchmark. Technical Report AEM-UoM-2003-1, Department of Aerospace and Engineering Mechanics, University of Minnesota 2003.

63. Mclean D. Automatic Flight Control Systems. Prentice-Hall, 1990. 